CNS Spectrums (2017), 22, 495-503. C Cambridge University Press 2017. This is an Open Access article, distributed under the terms of the Creative Commons Attribution licence (http://creativecommons.org/licenses/by /4.0/), which permits unrestricted re-use, distribution, and reproduction in any medium, provided the original work is properly cited.

\title{
Problematic internet use in gamblers: impact on clinical and cognitive measures
}

\author{
Samuel R. Chamberlain, ${ }^{1,2}$ Sarah A. Redden, ${ }^{3}$ Eric Leppink, ${ }^{3}$ and Jon E. Grant ${ }^{3 *}$
}

\footnotetext{
${ }^{1}$ Department of Psychiatry, University of Cambridge, Cambridge, UK

${ }^{2}$ Cambridge and Peterborough NHS Foundation Trust, Cambridge, UK

${ }^{3}$ Department of Psychiatry and Behavioral Neuroscience, University of Chicago, Illinois, USA
}

Objective. Gambling is a commonplace phenomenon, existing along a continuum from occasional gambling to functionally impairing gambling disorder. The internet may act as a conduit for some gambling behaviors. The impact of problematic internet use on clinical and cognitive features relevant to gambling has received little research attention.

Methods. A total of 206 adults aged 18-30 years who gamble at least five times per year were recruited from the general community and undertook detailed clinical and cognitive assessments. Problematic internet use was defined using a total score of 5 or more on Young's Diagnostic Questionnaire (YDQ). Linear regression was employed to evaluate the relative contribution of addictive-related, impulsive-related, and compulsive-related measures in predicting YDQ total scores in gamblers.

Results. Gamblers with problematic internet use (18\% of the sample) reported lower quality of life, lower self-esteem, elevated rates of intermittent explosive disorder, gambling disorder symptoms, attention deficit hyperactivity disorder (ADHD) symptoms, antisocial personality disorder, and posttraumatic stress disorder (PTSD), as well as relative deficits in decision making and spatial working memory. In linear regression, the extent of problematic internet use was most significantly associated with increased gambling disorder symptoms and increased ADHD symptoms.

Conclusions. Problematic internet use in gamblers is associated with worse quality of life, more problem/pathological gambling symptoms, more psychiatric morbidities, and select cognitive impairment. Refinement of the definition of problematic internet use and exploration of its clinical and cognitive associations are likely to be highly relevant to the treatment of problematic gambling.

Received 15 July 2016; Accepted 12 December 2016; First published online 12 September 2017

Key words: Compulsivity, impulsivity, cognition, internet, nosology, gambling.

\section{Introduction}

Despite being a recently developed technology, the internet is now all-pervasive in many parts of the world. In the United States, it is estimated that approximately $90 \%$ of adolescents and young adults use the internet, with high rates also being reported in Europe (e.g., the United Kingdom) and Asia. ${ }^{1,2}$ While internet use is less common in other parts of the world, its use is escalating

* Address correspondence and reprint requests to: Jon E. Grant, Department of Psychiatry and Behavioral Neuroscience, University of Chicago, Pritzker School of Medicine, 5841 South Maryland Avenue, MC 3077, Chicago, Illinois 60637.

(Email: jongrant@uchicago.edu) at a phenomenal rate, such as in Africa. There are clearly positive aspects of internet availability, including rapid access to global information sources and communication with others, but a proportion of individuals use the internet excessively, a putative entity referred to variably as "problematic internet use," "internet addiction," or "compulsive internet use." ${ }^{\prime 3}$ Indeed, the variety of terms pertaining to excessive use of the internet is indicative of a relative lack of consensus regarding optimal classification, and diagnosis, of such problems. Depending on the precise definition and population studied, prevalence estimates for problematic internet use vary from 1 to $35 \%$ in young people, being highest in Asia. ${ }^{2}$ Precise definition and nosological classification aside, excessive 
internet use has been clearly linked with untoward consequences, including worse academic achievement, ${ }^{4,5}$ greater risk of some mental disorders, ${ }^{6}$ and even-rarelysevere physical health consequences, such as deep-vein thrombosis due to prolonged periods of inactivity. ${ }^{7}$

Whether or not problematic internet use should constitute a formal mental disorder remains contentious. Some argue that excessive use of the internet is not a disorder in itself, but rather a consequence of, or incidental "through-route" for, other problems-such as compulsive sexual behavior (e.g., use of pornography), or compulsive shopping. ${ }^{42,43}$ Another area of debatewhich has influenced both the Diagnostic and Statistical Manual of Mental Disorders, 5th ed. (DSM-5) ${ }^{8}$ and the upcoming International Classification of Diseases, version 11 (ICD-11 $)^{9}$-examines how best to categorize problematic internet use.

The field of addiction research has increasingly considered whether certain behaviors have addictive "qualities." In the DSM-5, gambling disorder was incorporated into the broader category of "Substance-Addiction and Related Disorders" on the basis of evidence indicative of overlapping phenomenology, comorbidity, and neurobiology with substance use disorders. ${ }^{10-12}$ Various types of behavior have been posited as potential "behavioral addictions," including grooming (excoriation disorder, hair-pulling disorder), compulsive stealing (kleptomania), compulsive buying, compulsive sexual behavior, and problematic internet use. Some of these conditions are listed in the DSM-5 as impulse control disorders (e.g., kleptomania), and others as obsessive-compulsive-related disorders (excoriation disorder, hair-pulling disorder), while problematic internet use is listed only as a condition warranting further research. ${ }^{13}$ The Working Group for the International Classification of Diseases, version 11 (ICD11) argues for a broad category of impulse control disorders, including gambling disorder and compulsive sexual behavior, but, consistent with the DSM approach, considers that there are insufficient data to justify inclusion of problematic internet use as a formal entity. ${ }^{9}$ Alternative nosological approaches toward problematic internet use may include considering it an impulse control disorder or a compulsive disorder. Prevailing neurobiological models of problematic internet use highlight the likely contribution of cognitive dysfunction relating to impaired decision making (such as preference for more immediate reward), executive dysfunction (e.g., setshifting difficulties), and impaired impulse control. ${ }^{23,41}$. In the absence of a consensus as to which cognitive deficits are the most prominent in sufferers, these findings may be interpreted in support of any of the three nosological approaches (addictive, impulsive, or compulsive).

The aim of this study was twofold: first, to describe differences between gamblers with problematic internet use and gamblers without problematic internet use on demographic, clinical, and cognitive measures; and second, to evaluate, from these measures, those most strongly associated with problematic internet use severity in gamblers. We included structured screening assessments for mainstream mental disorders as well as impulse control disorders, along with quality-of-life and self-esteem questionnaires, plus objective neurocognitive assessment using tests that capture aspects of flexible responding, decision making, inhibitory control, and working memory. We hypothesized that problematic internet use in gamblers would be associated with a lower quality of life, lower self-esteem, and more severe gambling symptoms, consistent with the notion of it representing a psychiatric disorder of interest and relevance, and a potential "gateway" behavior for gambling. We further hypothesized that internet use disorder in gamblers would be most strongly predicted by impulsive measures, suggesting that it might best be conceptualized within this framework (rather than as a substance use and related addictive or compulsive [obsessive-compulsive] type of disorder).

\section{Method}

\section{Participants}

A total of 206 individuals aged 18-29 years were recruited using media advertisements in two major U.S. cities. The only inclusion criterion was gambling at least five times in the preceding year, as the study was part of a broader project examining gambling in young adults. The only exclusion criterion was an inability to understand/undertake the assessments. Participants provided written informed consent after receiving a complete description of the study and attended the study center to complete detailed questionnaires and undertake structured psychiatric interviews.

\section{Assessments}

Participants attended study centers in order to complete detailed demographic, clinical, and cognitive assessments. Validated screening tools for problematic internet use are relatively sparse and underresearched. Problematic internet use was quantified using Young's Diagnostic Questionnaire (YDQ). ${ }^{14}$ The YDQ is an eightitem yes/no questionnaire that was derived from prior criteria for substance use disorder and pathological gambling, but applied to maladaptive use of the internet. The instrument considers preoccupation with the internet, escalating quantities of time spent using the internet, repeated unsuccessful attempts to cut back, restlessness/ irritability when attempting to cut back, staying online longer than intended, jeopardizing careers/scholarship/ relationships, lying to others, and using the internet to 
escape from life or emotional difficulties. As such, we felt that the YDQ appropriately captured a broad range of problematic internet use thoughts and behaviors. Problematic internet addiction was defined as endorsing five or more of these symptoms over the preceding 12-month period, consistent with Young's original operational criteria. ${ }^{14}$

The presence of current psychiatric disorders was evaluated using the Mini International Neuropsychiatric Inventory (MINI) ${ }^{15}$ and the Minnesota Impulse Disorder Inventory (MIDI). ${ }^{16}$ The MINI identifies mainstream psychiatric disorders such as mood and anxiety disorders, while the MIDI identifies impulse control disorders. Quality of life was measured using the Quality of Life Inventory (QOLI), ${ }^{17}$ self-esteem with the Rosenberg Self-Esteem Scale (RSE), ${ }^{18}$ and emotional dysregulation with the Difficulties with Emotion Regulation Scale (DERS). ${ }^{19}$ To quantify impulsive and compulsive tendencies, participants completed the Padua ObsessiveCompulsive Inventory, ${ }^{20}$ the Adult ADHD Self-Report Scale (ASRS, v1.1, 2005), ${ }^{21}$ and the Structured Clinical Inventory for Pathological Gambling (SCI-PG) (modified for the DSM-5). ${ }^{22}$ We also collated information regarding frequency of alcohol use and number of cigarette packs smoked per day.

Cognitive testing was conducted using four paradigms from the Cambridge Neuropsychological Test Automated Battery (CANTABeclipse, v. 3, Cambridge Cognition Ltd., Cambridge, UK). Based on existing models of internet use disorder, ${ }^{23,41}$ we focused on top-down inhibitory control, decision making, working memory, and set-shifting.

Top-down inhibitory control was measured using the Stop Signal Task (SST). On the SST, a series of directional arrows was presented on the computer screen one at a time, and volunteers made quick responses depending on the direction of the arrows (left button for left-facing arrow; right button for right-facing arrow). On some trials, an auditory stop signal ("beep") occurred at a variable time after presentation of the "go" cue, indicating that the volunteer should attempt to omit a response for the given trial. By dynamically modulating the time between presentation of the arrow and the stop signal, the task calculated the stop-signal reaction timea measure of time taken to suppress a response that would normally be made. Longer stop-signal reaction times equate to worse top-down control.

Decision making was measured using the Cambridge Gambling Task (CGT). On each trial, 10 boxes were shown, some blue and some red, with a token having been hidden behind one of these. The participant selected the color of the box they believed a token was hidden behind and then decided how many points to gamble, having made the correct decision. The main measures of decision making on the task were the proportion of points gambled overall, the proportion of rational decisions made (proportion of trials when the volunteer opted for the color that was in the majority), and the extent of risk adjustment (the extent to which individuals modulated the amount gambled depending on the probability of making correct choices).

Spatial working memory was assessed using the spatial working memory task (SWM). Volunteers had to find "tokens" hidden behind boxes on the computer screen and avoid returning to boxes where tokens had previously been located. The key outcome measure from the task was the total number of errors made (inappropriately returning to boxes that had previously yielded tokens).

Set-shifting was assessed using the Intra-Dimensional/Extra-Dimensional Set-Shift task (IED). This paradigm is based on the Wisconsin Card Sorting Task but decomposes different aspects of rule acquisition and flexible responding over nine task stages. Volunteers choose between two stimuli presented on the computer screen on each trial and receive feedback as to whether their choice is "right" or "wrong." Through trial and error, the volunteer attempts to learn a rule for which a stimulus is correct. The computer alters this underlying rule when the current rule has been learned by the volunteer. The main measure on the task is the total number of errors made, adjusted for stages that were failed. Where this composite measure is statistically significant for a comparison of interest, then scores on individual task stages can be explored, to confirm the main cognitive problems driving overall impairment on the task.

\section{Data analysis}

Participants were grouped according to having internet addiction (Internet Addiction Questionnaire [IADQ] score of 5 or more) or not (reference group). The demographic, clinical, and cognitive characteristics of the two study groups were tabulated and compared using a one-way analysis of variance (ANOVA) or chi-squared $\left(\chi^{2}\right)$ tests. Yates's correction was applied where appropriate for the $\chi^{2}$ tests.

In order to identify variables that were most strongly associated with higher internet addiction scores (YDQ scores) in gamblers, variables related to impulsivity, compulsivity, and addiction that differed significantly between study groups were entered into linear regression (dependent variable $=$ YDQ total score) across the whole study sample, using the ENTER method ( $p$ for entry $<0.05$, for exit $>0.10$ ). Conformity to model assumptions was tested using the Durbin-Watson test, tolerance, colinearity diagnostics, residual statistics (Mahalanobis distance), and manual inspection of data plots (including residuals). 
Statistical significance was defined as $p<0.05$ uncorrected throughout. The data were analyzed using SPSS (v. 22; SPSS Inc., Chicago, IL, USA).

\section{Results}

Of the 206 participants enrolled into the study, 37 (18\% of the sample) met the criteria for problematic internet use based on the YDQ. The summative demographic, clinical, and cognitive characteristics of the two study groups are presented in Table 1, where it can be seen that the groups did not differ significantly in terms of age, gender, or levels of education.

Compared to gamblers without problematic internet use, gamblers with problematic internet use demonstrated significantly lower quality of life, lower self-esteem, more difficulties with emotional regulation, increased occurrence of mainstream mental disorders, increased occurrence of impulse control disorders, higher attention deficit hyperactivity disorder (ADHD) symptoms, and higher gambling disorder symptoms. The groups did not differ significantly in terms of alcohol or tobacco consumption, nor obsessive-compulsive scores. It can be seen that, in comparison to participants without problematic internet use, those with problematic internet use demonstrated significantly greater occurrence of posttraumatic stress disorder (PTSD), antisocial personality disorder, intermittent explosive disorder, and gambling disorder.

In terms of cognitive functioning, problematic internet use was associated with relative impairments in decision making (lower risk adjustment) and spatial working memory (more total errors) but did not differ from the reference group in terms of stop-signal inhibitory control or set-shifting.
Linear regression identified a statistically significant model that accounted for $19 \%$ of the variance in YDQ total scores across all gamblers, according to the $R^{2}$ statistic $(F=7.433, p<0.001)$. Durbin-Watson, colinearity diagnostics, tolerance, and residual criteria were within the thresholds for model validity. Variables that were individually significant predictors for YDQ total scores were SCI-GD total scores $(\beta$ standardized $=$ $0.070, t=4.133, p<0.001)$, and ADHD symptoms ( $\beta$ standardized $=0.212, t=3.161, p=0.002)$. Presence of current mainstream mental disorder (MINI), current impulse control disorder (MIDI), CGT risk adjustment, and SWM total errors were not statistically significant predictors in the model (all $p>0.20$ ). Casewise diagnostics identified two subjects who were outliers, and exclusion of their data did not qualitatively affect the results of the regression model. The model constant was significant $(t=3.105, p=0.002), \beta$ unstandardized = 1.137; see Tables 2-4).

\section{Discussion}

In the present study, we assessed the occurrence of problematic internet use in a non-treatment-seeking sample of young adults who gamble at least occasionally, recruited from U.S. settings. We also explored the relationships between problematic internet use and other variables germane to the debate about the potential impact of internet use on gambling.

\section{Problematic internet use and gambling behaviors}

The finding that problematic internet use was evident in $18 \%$ of gamblers suggests that this is a common issue for

TABLE 1. Overview of demographic and clinical characteristics in the study groups

\begin{tabular}{|c|c|c|c|c|c|c|c|}
\hline & \multicolumn{2}{|c|}{ Controls $(n=169)$} & \multicolumn{2}{|c|}{ Problematic internet use $(n=37)$} & \multicolumn{2}{|c|}{ ANOVA } & \\
\hline & Mean & $S D$ & Mean & $S D$ & $F$ & $p$ & \\
\hline Age, years & 22.99 & 3.72 & 24.14 & 3.47 & 2.870 & 0.092 & \\
\hline Gender, proportion of male subjects & 0.59 & & 0.53 & & $0.327^{@}$ & 0.568 & \\
\hline Education score & 3.36 & 0.81 & 3.36 & 0.72 & 0.002 & 0.967 & \\
\hline Quality-of-life $t$ score & 45.93 & 12.56 & 39.68 & 12.50 & 7.020 & 0.009 & $* *$ \\
\hline Alcohol consumption, drinks/week & 1.55 & 1.42 & 1.51 & 1.35 & 0.018 & 0.893 & \\
\hline Nicotine consumption, packs per day & 0.16 & 0.33 & 0.13 & 0.31 & 0.295 & 0.588 & \\
\hline Rosenberg Self-Esteem total score & 21.26 & 6.93 & 16.92 & 5.72 & 12.324 & $<0.001$ & $* * *$ \\
\hline Difficulties with emotion regulation total score & 73.50 & 19.99 & 84.28 & 22.68 & 8.193 & 0.005 & $* *$ \\
\hline Current mainstream psychiatric disorder, MINI, proportion of subjects & 0.43 & & 0.68 & & $7.229^{@}$ & 0.0072 & $* *$ \\
\hline Current impulse control disorder, MIDI, proportion of subjects & 0.11 & & 0.24 & & $4.423^{@}$ & 0.0355 & * \\
\hline Padua total score & 24.48 & 70.71 & 37.30 & 28.92 & 1.169 & 0.281 & \\
\hline ADHD symptoms (ASRS total score) & 8.79 & 5.22 & 11.16 & 5.64 & 6.082 & 0.014 & * \\
\hline SCI-GD total score & 1.34 & 1.87 & 3.08 & 3.22 & 19.323 & $<0.001$ & $* * *$ \\
\hline Body-mass index & 24.74 & 5.33 & 27.38 & 8.33 & 5.887 & 0.016 & * \\
\hline
\end{tabular}


TABLE 2. Proportion of subjects in each group with individual mainstream psychiatric disorders, as indexed by the Mini International Neuropsychiatric Inventory (MINI)

\begin{tabular}{|c|c|c|c|c|c|}
\hline & \multirow{2}{*}{$\begin{array}{c}\text { Controls }(n=169) \\
\text { Proportion }\end{array}$} & \multirow{2}{*}{$\begin{array}{c}\text { Problematic internet use }(n=37) \\
\text { Proportion }\end{array}$} & \multicolumn{2}{|c|}{$\chi^{2}$} & \\
\hline & & & $x$ & $p$ & \\
\hline Major depressive disorder & 0.095 & 0.176 & 1.923 & 0.166 & \\
\hline Manic episode & 0.110 & 0.057 & 0.475 & 0.4909 & \\
\hline Panic disorder & 0.027 & 0.034 & 0.007 & 0.933 & \\
\hline Agoraphobia & 0.070 & 0.147 & 0.911 & 0.340 & \\
\hline Social phobia & 0.042 & 0.139 & 3.301 & 0.069 & \\
\hline Obsessive-compulsive disorder & 0.024 & 0.057 & 0.208 & 0.649 & \\
\hline Posttraumatic stress disorder & 0.006 & 0.081 & 5.492 & 0.0191 & * \\
\hline Alcohol use disorder, any & 0.168 & 0.306 & 3.426 & 0.0642 & \\
\hline Substance use disorder, any & 0.143 & 0.152 & 0.012 & 0.913 & \\
\hline Anorexia & 0.006 & 0.027 & 0.068 & 0.794 & \\
\hline Bulimia & 0.024 & 0.057 & 0.208 & 0.649 & \\
\hline General anxiety disorder & 0.054 & 0.111 & 0.822 & 0.365 & \\
\hline Antisocial personality disorder & 0.077 & 0.222 & 6.433 & 0.011 & * \\
\hline
\end{tabular}

TABLE 3. Proportion of subjects in each group with individual impulse control disorders, as measured using the Minnesota Impulse Disorder Inventory (MIDI)

\begin{tabular}{|c|c|c|c|c|c|}
\hline & \multirow{2}{*}{$\frac{\text { Controls }(n=169)}{\text { Proportion }}$} & \multirow{2}{*}{$\frac{\text { Problematic internet use }(n=37)}{\text { Proportion }}$} & \multicolumn{2}{|c|}{$\chi^{2}$} & \\
\hline & & & $X$ & $p$ & \\
\hline Compulsive buying disorder, MIDI & 0.024 & 0.108 & 3.757 & 0.0526 & \\
\hline Kleptomania, MIDI & 0.000 & 0.000 & n/a & $\mathrm{n} / \mathrm{a}$ & \\
\hline Trichotillomania, MIDI & 0.000 & 0.000 & n/a & $\mathrm{n} / \mathrm{a}$ & \\
\hline Intermittent explosive disorder, MIDI & 0.018 & 0.139 & 8.281 & 0.004 & $* *$ \\
\hline Pyromania, MIDI & 0.006 & 0.000 & n/a & $\mathrm{n} / \mathrm{a}$ & \\
\hline Gambling disorder, MIDI & 0.216 & 0.432 & 7.241 & 0.0071 & ** \\
\hline Compulsive sexual behavior, MIDI & 0.042 & 0.054 & 0.116 & 0.734 & \\
\hline Binge-eating disorder, MIDI & 0.036 & 0.027 & 0.066 & 0.797 & \\
\hline Skin-picking disorder, MIDI & 0.034 & 0.107 & 2.071 & 0.150 & \\
\hline
\end{tabular}

\section{TABLE 4. Cognitive performance in the study groups}

\begin{tabular}{|c|c|c|c|c|c|c|c|}
\hline & \multicolumn{2}{|c|}{ Controls $(n=169)$} & \multicolumn{2}{|c|}{ Problematic internet use ( $n=37)$} & \multicolumn{2}{|c|}{ ANOVA } & \\
\hline & Mean & $S D$ & Mean & $S D$ & $F$ & $p$ & \\
\hline SST/SSRT (last half) & 185.85 & 76.57 & 203.64 & 74.69 & 1.648 & 0.201 & \\
\hline CGT overall proportion bet & 0.54 & 0.16 & 0.59 & 0.13 & 3.376 & 0.068 & \\
\hline CGT quality of decision making & 0.93 & 0.11 & 0.91 & 0.11 & 1.062 & 0.304 & \\
\hline CGT risk adjustment & 1.29 & 1.25 & 0.81 & 1.05 & 4.755 & 0.030 & * \\
\hline SWM total errors & 21.39 & 20.82 & 32.76 & 22.28 & 8.810 & 0.003 & ** \\
\hline
\end{tabular}

CGT = Cambridge Gambling Task; IED = Intra-Dimensional/Extra-Dimensional set-shifting; SSRT = stop-signal reaction time; SST = stop-signal test; SWM $=$ spatial working memory task.

${ }^{*} p<0.05 ;{ }^{* *} p<0.01$.

gamblers, rather than a rarity or an entity that is only of academic interest. Rates may well be higher in psychiatric inpatient and outpatient populations of people with gambling problems. The global prevalence of problematic internet use is likely to grow over time, along with increasing availability of the internet. The prevalence rate of problematic internet use seen here is comparable or only slightly higher as compared to other 
studies conducted in U.S. settings, which found prevalence ranging from 9.8 to $15.2 \%$ in young people. ${ }^{1}$ However, our sample focused on people who gamble at least five times a year, and so our findings may well not be generalizable to the background population.

Additionally, we found that problematic internet use in gamblers was associated with a quality of life that was significantly impaired (in the "poor range," akin to that previously reported in depression ${ }^{25}$ ), with associated concomitant impairment in terms of self-esteem. These findings are consistent with previous research that found low levels of self-esteem in people with excessive internet use, especially in those with more severe symptoms. ${ }^{26}$

It has been argued that problematic internet use may represent an avenue through which individuals undertake specific types of repetitive, addictive, or compulsive acts, rather than being an independent disorder. Our data highlight that problematic use of the internet is associated with higher rates of problem and pathological gambling symptoms among people who gamble, highlighting the internet as a key "gateway" through which gambling problems can manifest. Using gold-standard inperson clinical instruments, we found that the majority of cases of problematic internet use in gamblers were not associated with another type of impulse control disorder that would better account for the symptoms, including formal gambling disorder, in our sample. Thus, it appears that problematic internet use can contribute to worse gambling but does not lead to pathological gambling in all people. Nonetheless, rates of gambling disorder were significantly higher in problematic internet users, and rates of compulsive sexual behavior and compulsive shopping disorder were numerically higher but not statistically significantly higher versus the reference group. Furthermore, extent of problem gambling was a significant predictor of the extent of problematic internet use in our regression modeling.

\section{How best should problematic internet use be conceptualized?}

Optimal nosological classification of any psychiatric disorder is informed by understanding of etiology, comorbidity, and neurobiology. ${ }^{24}$ Prevailing suggestions as to the preferred classification of problematic internet use include as a "behavioral addiction," as an obsessivecompulsive (or, simply, compulsive) disorder, or as an impulse control disorder. ${ }^{28,29}$ The current study was not designed to address etiology and therefore considered comorbidity and neurobiology in turn.

In an extensive review, Ko and colleagues ${ }^{26}$ reported that internet addiction has most frequently been associated, in the existing literature, with $\mathrm{ADHD}$, depression, social anxiety disorder, substance use disorder, and aggressive behaviors (for discussion, see also $^{2}$ ). There are limitations in many of the available studies, such as the use of non-gold-standard instruments to assess the presence or absence of disorders, and frequent failure to screen for impulse control disorders at all.

The concept of problematic internet use as a form of behavioral addiction, sharing parallels with substance addiction, is central to several working definitions of the disorder. In the current study, we used diagnostic criteria that were derived from modeling gambling disorder and substance use disorder. ${ }^{14}$ Contrary to our expectations, we did not find any significant associations between problematic internet use and substance use disorders, based on careful structured clinical assessments, nor were significant relationships evident between occurrence of problematic internet use and self-reported frequencies of alcohol and nicotine consumption.

People with problematic internet use did not show higher rates of formal obsessive-compulsive disorder (OCD; by structured clinical interview), nor did they exhibit higher-than-expected levels of obsessive compulsiveness as indexed by the self-complete Padua Inventory, which was developed to assess obsessive-compulsive (OC) traits in normative populations. ${ }^{20}$

We did, however, find evidence in support of problematic internet use being related to the classically recognized impulse control disorders, to problem gambling, and to ADHD. Overall presence of one or more impulse control disorders was significantly higher in problematic internet users than in controls, as was the extent of problem gambling as indexed by the SCI-GD. When individual impulse control disorders were considered, structured clinical assessment identified significantly higher rates of gambling disorder and intermittent explosive disorder in problematic internet users. Antisocial personality disorder was also more common in cases than in controls. Furthermore, self-rated maladaptive gambling and ADHD symptom scores were statistically significant predictors of the extent of problem internet use on conservative regression modeling.

Cognitive domains potentially implicated in the pathophysiology of problematic internet use include failures of top-down inhibitory control, working memory, and decision making (for review, see Brand et al. ${ }^{23}$ ). It should be noted that many cognitive and imaging studies in this field have focused on internet gaming addiction, rather than problematic internet use per se. Only a handful of studies have addressed cognitive functioning in problematic internet users. ${ }^{23,41}$

In gamblers, we identified an association between problematic internet use and impaired spatial working memory, along with a nonglobal deficit in decision making (less modulation of behavior as a function of gambling risk level), but intact top-down response suppression and set-shifting. Other studies that did not recruit participants on the basis of gambling symptoms are informative. One such prior study examined working 
memory in problematic internet users, albeit using a digit-span task, and found deficits. ${ }^{30}$ Other studies have reported deficits in decision making using the Iowa Gambling Task and the Game of Dice Task. ${ }^{31,32}$ Two of three previous studies found intact top-down inhibitory control in problematic internet users versus controls, albeit using go/no-go rather than stop-signal tests (intact, ${ }^{31,33}$ impaired ${ }^{30}$ ). As such, our data are broadly consistent with the majority of the small volume of existing literature, in that similarly affected cognitive domains were found.

It is potentially informative to qualitatively compare the profile of neuropsychological impairment observed here in problematic internet users to findings using the same computerized cognitive tasks in impulse control, addictive, and obsessive-compulsive disorders. Caution is needed because other studies did not focus on gamblers per se. Interestingly, impaired working memory and risk adjustment, but intact response inhibition, were previously found in compulsive buying disorder ${ }^{34}$ and in nicotine consumers. ${ }^{35}$ Other conditions appeared to be associated with more global decision-making deficits, notably shoplifting, ${ }^{36}$ and problem gambling with a history of trauma. ${ }^{37}$ For OCD, decision making was generally found to be intact while response inhibition was not. ${ }^{38,39}$ In all, the current cognitive data suggest similarities between problematic internet use and some addictions and impulse control disorders, rather than with OCD. It is important to note that similar behavioral deficits across disorders could be driven by different neurobiological abnormalities. Functional neuroimaging work could help to address the neurobiological relationships between problematic internet use and other disorders.

\section{Limitations of the Study}

This study was undertaken in non-treatment-seeking young adults recruited from U.S. settings who gambled at least five times in the preceding year, and so our findings may not generalize to other populations, such as older individuals, people who do not gamble so frequently, or those presenting to mental health services. This being an exploratory study, we did not correct for multiple comparisons. Nonetheless, the relationship among problematic internet use, gambling symptoms, and ADHD symptoms was robust in a secondary linear regression analysis, even accounting for all other variables. We used the total score from Young's Diagnostic Questionnaire to assess the extent of problematic internet use, an instrument that is brief and therefore convenient. We believe this to be a reasonable instrument, as it is largely equivalent to the widely accepted SCI-PG, used to assess problematic gambling behavior, in terms of the overarching symptom domains considered. Nonetheless, the
YDQ may suffer from a tendency to over-pathologize, and also lacks key items related to internet gaming disorder criteria and other nosological approaches. The threshold used on the YDQ for "problematic internet use" requires further validation in future work. Nonetheless, we feel that the currently used threshold was consistent with other disorder criteria (gambling disorder, substance use disorder); furthermore, it did identify participants with impaired quality of life versus the reference group. Also, we used a complementary approach of linear regression to examine associations between total YDQ criteria scores and other measures, which confirmed an association with worse gambling symptoms and more ADHD symptoms. The field would benefit from future research to assess the validity and optimal type of symptom scale for internet addiction. Because the YDQ yields a total score in the range of 0 to 8 , this would have limited the available variance in the regression modeling with respect to the dependent variable. Due to this issue and the sample size, subtler relationships between problematic internet use and other measures may have been overlooked.

\section{Conclusions}

The current findings demonstrate an association between problematic internet use and impaired quality of life in gamblers, suggesting that it is an entity that merits further clinical and research attention. In our study, problematic internet use appears to be more closely related, in terms of comorbidity, with classic impulse control disorders, and with ADHD, versus substance addiction, mood disorders, or OCD (including obsessive-compulsive personality traits as indexed by the Padua Inventory). As expected, problematic internet use was associated with worse gambling symptoms in our sample, supporting the notion of the internet as a "gateway" through which one might engage in rewarding behaviors. These findings add initial weight to one perspective-namely, that problematic internet use might be considered more of an impulse control disorder or gambling-related disorder than an obsessive-compulsive or substance addiction and substance-related disorder. ${ }^{40}$ There are various criteria that can be used to assess whether a particular set of symptoms constitutes a meaningful or cohesive psychiatric disorder for inclusion in diagnostic nosological systems. Working groups for both the DSM-5 and ICD-11 considered problematic internet use, concluding that there was insufficient data for formal inclusion but highlighting the need for more research. ${ }^{8,9}$ Inclusion of a disorder should be done on the grounds of clinical utility and diagnostic validity. ${ }^{24}$ Findings from the current study help to inform this ongoing debate by highlighting the salience of problematic internet use for understanding the clinical and 
cognitive presentations observed in gamblers. Problematic use of the internet may constitute a candidate treatment "target" in the management of gambling disorder for a subset of patients.

\section{Disclosures}

Dr. Grant reports grants from the National Center for Responsible Gaming during the conduct of the study, and grants from Forest Pharmaceuticals, Takeda, Brainsway, and Roche, and others from Oxford Press, Norton, McGraw-Hill, and American Psychiatric Publishing outside of the submitted work.

Dr. Chamberlain reports personal fees from Cambridge Cognition outside of the submitted work. Dr. Chamberlain's research is funded by a clinical fellowship from the Wellcome Trust (grant reference number 110049/Z/15/Z).

Sarah Redden and Eric Leppink hereby state that have nothing to disclose.

\section{REFERENCES:}

1. Moreno MA, Jelenchick L, Cox E, Young H, Christakis DA. Problematic internet use among US youth: a systematic review. Arch Pediatr Adolesc Med. 2011; 165(9): 797-805. Epub ahead of print May 2. https://www.ncbi.nlm.nih.gov/pmc/articles/PMC3215336/ pdf/nihms328899.pdf. Accessed January 15, 2017.

2. Spada MM. An overview of problematic internet use. Addict Behav. 2014; 39(1): 3-6. Epub ahead of print Oct, 2013.

3. Shaw M, Black DW. Internet addiction: definition, assessment, epidemiology and clinical management. CNS Drugs. 2008; 22(5): 353-365.

4. Black DW, Belsare G, Schlosser S. Clinical features, psychiatric comorbidity, and health-related quality of life in persons reporting compulsive computer use behavior. J Clin Psychiatry. 1999; 60(12): 839-844.

5. Derbyshire KL, Lust KA, Schreiber LR, et al. Problematic internet use and associated risks in a college sample. Compr Psychiatry. 2013; 54(5): 415-422. Epub ahead of print Jan 9.

6. Chen SY, Tzeng JY. College female and male heavy internet users' profiles of practices and their academic grades and psychosocial adjustment. CyberPsychol Behav Soc Netw. 2010; 13(3): 257-362.

7. Braithwaite I, Maxwell G, Sparks S, Beasley R, Shirtcliffe P. A man who collapsed after using the internet. JRSM Open. $2014 ; 5(9)$ : 2054270414543397. https://www.ncbi.nlm.nih.gov/pmc/articles/ PMC4207275/. Accessed January 15, 2017.

8. American Psychiatric Association. Diagnostic and Statistical Manual of Mental Disorders, 5th ed. Washington, DC: American Psychiatric Association; 2013.

9. Grant JE, Atmaca M, Fineberg NA, Fontenelle LF, et al. Impulse control disorders and "behavioural addictions" in the ICD-11. World Psychiatry. 2014; 13(2): 125-127. https://www.ncbi.nlm. nih.gov/pmc/articles/PMC4102276/. Accessed January 15, 2017.

10. Romanczuk-Seiferth N, van den Brink W, Goudriaan AE. From symptoms to neurobiology: pathological gambling in the light of the new classification in DSM-5. Neuropsychobiology. 2014; 70(2): 95-102. Epub ahead of print Oct 30.

11. Blanco C, García-Anaya M, Wall M, et al. Should pathological gambling and obesity be considered addictive disorders? A factor analytic study in a nationally representative sample. Drug Alcohol Depend. 2015; 150: 129-134. Epub ahead of print Feb 25. https:// www.ncbi.nlm.nih.gov/pmc/articles/PMC4387020/. Accessed January 15, 2017.

12. Grant JE, Chamberlain SR. Gambling disorder and its relationship with substance use disorders: implications for nosological revisions and treatment. Am J Addict. 2015; 24(2): 126-131.

13. Petry NM, O'Brien CP. Internet gaming disorder and the DSM-5. Addiction. 2013; 108(1): 1186-1187. Epub ahead of print May 13. http://onlinelibrary.wiley.com/doi/10.1111/add.12162/pdf. Accessed January 15, 2017.

14. Young KS. Internet addiction: the emergence of a new clinical disorder. CyberPsychol Behav. 1998; 1: 237-244. http:// chabad4israel.org/tznius4israel/newdisorder.pdf. Accessed January 15, 2017.

15. Sheehan DV, Lecrubier Y, Sheehan KH, et al. The Mini-International Neuropsychiatric Interview (M.I.N.I.): the development and validation of a structured diagnostic psychiatric interview for DSM-IV and ICD-10. J Clin Psychiatry. 1998; 59(Suppl. 20): 22-33; quiz 34-57.

16. Grant JE. Impulse Control Disorders: A Clinician's Guide to Understanding and Treating Behavioral Addictions. New York: W.W. Norton \& Company; 2008.

17. Frisch MB, Cornell J, Villanueva M, Retzlaff PJ. Clinical validation of the Quality of Life Inventory: a measure of life satisfaction for use in treatment planning and outcome assessment. Psychol Assess. 1993; 4(1): 92-101.

18. Rosenberg M. Society and the Adolescent Self-Image. Princeton, NJ: Princeton University Press; 1965. http://science.sciencemag.org/ content/148/3671/804. Accessed January 15, 2017.

19. Fowler JC, Charak R, Elhai JD, Allen JG, Frueh BC, Oldham JM. Construct validity and factor structure of the difficulties in the Emotion Regulation Scale among adults with severe mental illness. J Psychiatr Res. 2014; 58: 175-180. Epub ahead of print Aug 10.

20. Sanavio E. Obsessions and compulsions: the Padua Inventory. Behav Res Ther. 1988; 26(2): 169-177.

21. Kessler RC, Adler L, Ames M, et al. The World Health Organization Adult ADHD Self-Report Scale (ASRS): a short screening scale for use in the general population. Psychol Med. 2005; 35(02): 245-256.

22. Grant JE, Leppink EW, Redden SA, Odlaug BL, Chamberlain SR. COMT genotype, gambling activity, and cognition. J Psychiatr Res. 2015; 68: 371-376. Epub ahead of print May 9.

23. Brand M, Young KS, Laier C. Prefrontal control and internet addiction: a theoretical model and review of neuropsychological and neuroimaging findings. Front Hum Neurosci. 2014; 8: 375. https:// www.ncbi.nlm.nih.gov/pmc/articles/PMC4034340/pdf/fnhum-0800375.pdf. Accessed January 15, 2017.

24. Regier DA, Kuhl EA, Kupfer DJ. The DSM-5: classification and criteria changes. World Psychiatry. 2013; 12(2): 92-98. https:// www.ncbi.nlm.nih.gov/pmc/articles/PMC3683251/pdf/wps00120092.pdf. Accessed January 15, 2017.

25. McAlinden NM, Oei TP. Validation of the Quality of Life Inventory for patients with anxiety and depression. Compr Psychiatry. 2006; 47(4): 307-314. Epub ahead of print Apr 19.

26. Ko CH, Yen JY, Yen CF, Chen CS, Chen CC. The association between internet addiction and psychiatric disorder: a review of the literature. Eur Psychiatry. 2012; 27(1): 1-8. Epub ahead of print Dec 6, 2011.

27. Vajda A, Láng A, Péley B. Investigation of the compulsive and impulsive behavioral addictions among adolescents [in Hungarian]. Psychiatr Hung. 2014; 29(2): 152-157.

28. Bernardi S, Pallanti S. Internet addiction: a descriptive clinical study focusing on comorbidities and dissociative symptoms. Compr Psychiatry. 2009; 50(6): 510-516. Epub ahead of print Jan 20. 
29. Shapira NA, Goldsmith TD, Keck PE Jr, Khosla UM, McElroy SL. Psychiatric features of individuals with problematic internet use. J Affect Disord. 2000; 57(1-3): 267-272.

30. Zhou Z, Zhou H, Zhu H. Working memory, executive function and impulsivity in internet-addictive disorders: a comparison with pathological gambling. Acta Neuropsychiatr. 2015; 24: 1-9. Epub ahead of print Sep 24, 2015.

31. Sun DL, Chen ZJ, Ma N, Zhang XC, Fu XM, Zhang DR. Decisionmaking and prepotent response inhibition functions in excessive internet users. CNS Spectr. 2009; 14(2): 75-81.

32. Pawlikowski M, Brand M. Excessive internet gaming and decision making: do excessive World of Warcraft players have problems indecision making under risky conditions? Psychiatry Res. 2011; 188(3): 428-433. Epub ahead of print Jun 17.

33. Dong G, Zhou H, Zhao X. Impulse inhibition in people with internet addiction disorder: electrophysiological evidence from a Go/ No-Go study. Neurosci Lett. 2010; 485(2): 138-142. Epub ahead of print Sep 15.

34. Derbyshire KL, Chamberlain SR, Odlaug BL, Schreiber LR, Grant JE. Neurocognitive functioning in compulsive buying disorder. Ann Clin Psychiatry. 2014; 26(1): 57-63.

35. Chamberlain SR, Odlaug BL, Schreiber LR, Grant JE. Association between tobacco smoking and cognitive functioning in young adults. Am J Addict. 2012; 21 (Suppl. 1): S14-S19.

36. Grant JE, Chamberlain SR, Schreiber LR, Odlaug BL. Neurocognitive deficits associated with shoplifting in young adults. Compr Psychiatry. 2012; 53(8): 1049-1055. Epub ahead of print Jun 6.
37. Leppink EW, Grant JE. Traumatic event exposure and gambling: associations with clinical, neurocognitive, and personality variables. Ann Clin Psychiatry. 2015; 27(1): 16-24.

38. Chamberlain SR, Fineberg NA, Menzies LA, et al. Impaired cognitive flexibility and motor inhibition in unaffected first-degree relatives of patients with obsessive-compulsive disorder. Am J Psychiatry. 2007; 164(2): 335-338. https://www.ncbi.nlm.nih.gov/ pmc/articles/PMC1892796/pdf/nihms-491.pdf. Accessed January $15,2017$.

39. Morein-Zamir S, Papmeyer M, Pertusa A, et al. The profile of executive function in OCD hoarders and hoarding disorder. Psychiatry Res. 2014; 215(3): 659-667. Epub ahead of print Dec 21, 2013. https://www.ncbi.nlm.nih.gov/pmc/articles/PMC3988927/ pdf/main.pdf. Accessed January 15, 2017.

40. Shapira NA, Lessig MC, Goldsmith TD, et al. Problematic internet use: proposed classification and diagnostic criteria. Depress Anxiety. 2003; 17(4): 207-216.

41. Dong G, Potenza MN. A cognitive-behavioral model of internet gaming disorder: theoretical underpinnings and clinical implications. J Psychiatr Res. 2014; 58: 7-11. Epub ahead of print Jul 17. https://www.ncbi.nlm.nih.gov/pmc/articles/PMC4448942/ pdf/nihms693963.pdf. Accessed January 15, 2017.

42. King D, Delfabbro P, Griffiths M. The convergence of gambling and digital media: implications for gambling in young people. J Gambl Stud. 2010; 26(2): 175-187. Epub ahead of print Sep 16, 2009.

43. Griffiths MD, Parke J. Adolescent gambling on the internet: a review. Int J Adolesc Med Health. 2010; 22(1): 59-75. 